University of South Carolina

Scholar Commons

1995

\title{
Proton Diffusion in Nickel Hydroxide Films: Measurement of the Diffusion Coefficient as a Function of State of Charge
}

\author{
Sathya Motupally \\ University of South Carolina - Columbia \\ Christopher C. Streinz \\ University of South Carolina - Columbia \\ John W. Weidner \\ University of South Carolina - Columbia, weidner@engr.sc.edu
}

Follow this and additional works at: https://scholarcommons.sc.edu/eche_facpub

Part of the Chemical Engineering Commons

\section{Publication Info}

Journal of the Electrochemical Society, 1995, pages 1401-1408.

(c) The Electrochemical Society, Inc. 1995. All rights reserved. Except as provided under U.S. copyright law, this work may not be reproduced, resold, distributed, or modified without the express permission of The Electrochemical Society (ECS). The archival version of this work was published in the Journal of the Electrochemical Society. http://www.electrochem.org/

Publisher's link: http://dx.doi.org/10.1149/1.2048589

DOI: $10.1149 / 1.2048589$

This Article is brought to you by the Chemical Engineering, Department of at Scholar Commons. It has been accepted for inclusion in Faculty Publications by an authorized administrator of Scholar Commons. For more information, please contact digres@mailbox.sc.edu. 


\section{REFERENCES}

1. E.g. Lawrence and E. Tannas, Jr., Flat-Panel Displays and CRTs, Van Nostrand Reinhold Company, New York (1985).

2. T. Shinoda, M. Wakitani, T. Nanto, K. Yoshikawa, A Ohtsuka, and T. Hirose, SID '93 Digest, 14-1, 161 (1993).

3. K. Machida, H. Uchiike, H. Sasaki, and E. Munemoto, Proc. Japan Display'92, S16-4, 613 (1992).

4. A. Manabe, H. Uchiike, K. Machida, K. Baba, M. Tokura, and K. Amano, SID '93 Digest, P-40, 592
(1993).

5. T. Kanehara and I. Koiwa, Proc. 1994 IEICE Spring Conference, C-551, 5-118 (1994).

6. A. Nishida, K. Yoshida, H. Igarashi, and W. Kobayashi, Advances in Ceramics, Vol. 21: Ceramic Powder Science, p. 271, American Ceramic Soc. Inc., Westerville, $\mathrm{OH}(1987)$.

7. I. Koiwa, and H. Sawai, J. Inst. Television Engineers Jpn., 47, 1006 (1993).

8. K. Machida, K. Amano, M. Tokura, and H. Uchiike, ITEJ Technical Report, 17, 25 (1993).

\title{
Proton Diffusion in Nickel Hydroxide Films
}

\section{Measurement of the Diffusion Coefficient as a Function of State of Charge}

\author{
Sathya Motupally, ${ }^{*}$ Christopher C. Streinz, and John W. Weidner** \\ Department of Chemical Engineering, University of South Carolina, Columbia, South Carolina 29208
}

\section{ABSTRACT}

Electrochemical impedance spectroscopy (EIS) was used to measure the solid-state diffusion coefficient of protons in nickel hydroxide films at room temperature as a function of state of charge (SOC). A model for the complex faradaic impedance of the nickel hydroxide active material is presented and used to extract the diffusion coefficient of protons from the EIS data. Impedance data over a range of frequencies can be used to extract a constant diffusion coefficient without the knowledge of the initial mobile proton concentration or the form of the charge-transfer kinetic expression. The proton diffusion coefficient is a strong function of SOC and decreases approximately three orders of magnitude from $3.4 \times 10^{-8}$ to $6.4 \times 10^{-11} \mathrm{~cm}^{2} \mathrm{~s}^{-1}$ as the electrode discharges from the completely charged to the completely discharged state. The measurements were performed on well-conditioned nickel hydroxide films and therefore it is likely that the diffusion coefficients measured correspond to the $\beta$-phase of the active material. The diffusion coefficient of protons was measured for three different film thicknesses, 1.5, 1.2, and $1.0 \mu \mathrm{m}$. The diffusion coefficient is independent of the thickness of the film as predicted by theory. The three orders of magnitude decrease in the diffusion coefficient of protons can be explained on the assumption that the protons move predominantly through the oxidized phase [NiOOH] which is interdispersed along with the reduced phase $\left[\mathrm{Ni}(\mathrm{OH})_{2}\right]$ in the active material.

\section{Introduction}

The nickel hydroxide electrode is the positive plate of many rechargeable battery systems such as the nickel/cadmium, nickel/hydrogen, and nickel/metal hydrides. The redox reaction taking place at the electrode during discharge and charge can be represented as

$$
\mathrm{NiOOH}+\mathrm{H}_{2} \mathrm{O}+e^{-} \underset{\text { charge }}{\stackrel{\text { discharge }}{\rightleftarrows}} \mathrm{Ni}(\mathrm{OH})_{2}+\mathrm{OH}^{-}
$$

It has been proposed that both charge and discharge are controlled by the solid-state diffusion of protons. ${ }^{1-10}$ MacArthur ${ }^{1}$ and Zimmerman and Effa ${ }^{2}$ proposed that during discharge a proton diffuses from the film/electrolyte interface into the active material and an electron enters across the conducting substrate/film interface. During charge the proton diffuses to the film/electrolyte interface to react with a hydroxyl ion to form water. A schematic diagram of a nickel hydroxide film along with the charge/ discharge mechanisms is depicted in Fig. 1. Mao et al. ${ }^{7}$ and Weidner and Timmerman ${ }^{8}$ modeled the discharge behavior of the nickel hydroxide electrode and reported that the polarization losses due to diffusion of protons is a critical factor in correlating the percent utilization of the active material to the discharge rate. Those authors concluded that a key parameter required to predict the discharge characteristics of the electrode quantitatively is the diffusion coefficient of protons through the active material.

Several researchers have reported data on the solid-state diffusion coefficient of protons in the nickel hydroxide active material using different electrochemical techniques. ${ }^{1,5,6,9,10}$ Great discrepancies, however, exist in the reported values of the proton diffusion coefficient from different sources. MacArthur ${ }^{1}$ and Zhang and Park ${ }^{6}$ extracted

\footnotetext{
* Electrochemical Society Student Member.
}

* Electrochemical Society Active Member. a diffusion coefficient of $1.9 \times 10^{-9}$ and $1.0 \times 10^{-11} \mathrm{~cm}^{2} \mathrm{~s}^{-1}$, respectively, from cyclic voltammetry studies. MacArthur assumed the charge/discharge reaction to be reversible whereas Zhang and Park assumed the reaction to be irreversible. MacArthur, ${ }^{9}$ Briggs and Snodin, ${ }^{5}$ and Zhang and Park $^{6}$ used chronoamperometry and reported values of $4.6 \times 10^{-11}, 1.0 \times 10^{-12}$, and $5.0 \times 10^{-12} \mathrm{~cm}^{2} \mathrm{~s}^{-1}$, respectively for the diffusion coefficient of protons, assuming reversible kinetics. Fan ${ }^{10}$ measured a diffusion coefficient ranging from $2.0 \times 10^{-8}$ to $5.0 \times 10^{-8} \mathrm{~cm}^{2} \mathrm{~s}^{-1}$ using chronoamperometry and fitted his data to a more realistic finite reaction rate-diffusion model.

Lukovtsev and Slaidin ${ }^{4}$ investigated the effect of the SOC of the electrode on the diffusion rate of protons by polarizing two sides of a nickel foil, anodically on one side and cathodically on the other, in $\mathrm{KOH}$. With respect to reaction 1 , the SOC of the electrode is defined as the fraction of $\mathrm{NiOOH}$ in the active material. They reported that the diffusion rate of the protons increases with the increase in the SOC of the electrode. However their measurements were only qualitative and did not include quantifying the value of the diffusion coefficient or the diffusion rate of the protons. If the diffusion rate of protons is a function of SOC, the reported values of the diffusion coefficient may correspond to different states of charge depending on the potential range in which the electrode was perturbed during the potential step experiments. In this paper, we report our findings on the measurement of the proton diffusion coefficient as a function of SOC using EIS. EIS is well suited for this work because it is a steady-state technique capable of holding the electrode at a constant SOC during the measurement.

Previous researchers used EIS to measure the solid-state diffusion coefficient of mobile species in electrochromic and polymeric films. ${ }^{11-20}$ Several methods are reported in the literature by which one can extract the solid-state diffusion coefficient of the diffusing species from EIS data. 


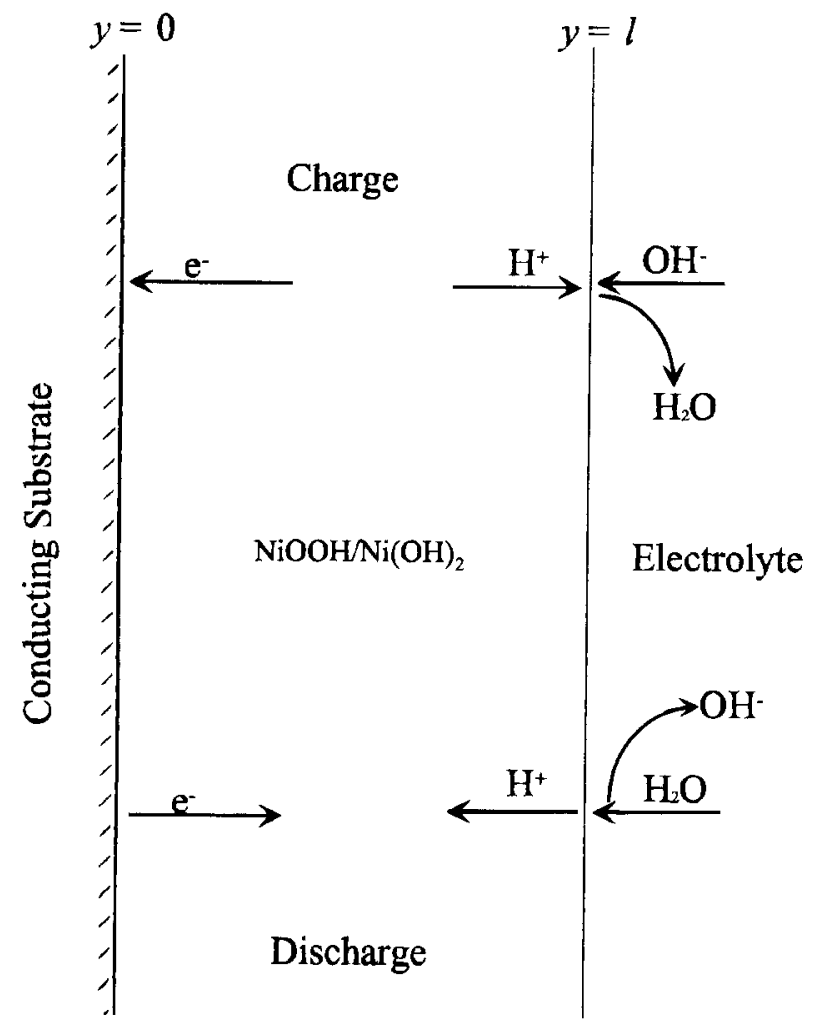

Fig. 1. A schematic diagram of the nickel hydroxide film along with the charge/discharge mechanism is depicted. The film with a thickness of $l$ is assumed to be uniformly deposited onto a conducting substrate. During discharge, a proton diffuses from the film/electrolyte interface into the active material and an electron enters across the conducting substrate/film interface. During charge, the proton diffuses to the film/electrolyte interface to react with a hydroxyl to form water.

One method is to use the data on the $45^{\circ}$ line seen on a Nyquist plot at high frequencies due to semi-infinite diffusion control (Warburg region). For example, this method was used by Ho et al. ${ }^{11}$ and Armstrong et al. ${ }^{12}$ to extract diffusion coefficients of $\mathrm{Li}^{+}$in $\mathrm{WO}_{3}$ and $\mathrm{IrCl}_{6}^{-2}$ in poly(4vinyl pyridine), respectively. The real or the imaginary components of the complex impedance in the Warburg region are plotted vs. the inverse of the square root of the angular frequency and the slope of the straight line obtained is substituted into the expression for the Warburg coefficient. The quantity that results from the analysis of the Warburg region is $C^{0} \sqrt{D}$, where $C^{0}$ is the concentration of the mobile species at the beginning of the EIS experiment and $D$ is the diffusion coefficient of the mobile species. To extract the diffusion coefficient, knowledge of $C^{0}$ is required, but because $C^{0}$ is not generally known with precision, the quantity $C^{0} \sqrt{D}$ is usually not separated into the constituent individual components. ${ }^{17,13} \mathrm{~A}$ different approach, also reported in the literature, is to use the low frequency capacitance and resistance to extract the diffusion coefficient. ${ }^{11,18}$ The diffusion coefficient is calculated from the equation $R_{\mathrm{L}} C_{\mathrm{L}}=l^{2} / 3 D$, where $R_{\mathrm{L}}$ is the low frequency resistance, $C_{\mathrm{L}}$ the low frequency capacitance, and $l$, the thickness of the film. This method cannot be used if there is an overlap between the charge-transfer and diffusion-controlled regions because of the uncertainty in the estimation of $R_{\mathrm{L}}$ and $C_{\mathrm{L}} \cdot{ }^{11,18}$ Another method propounded by Armstrong ${ }^{16}$ and discussed by Mathias and $\operatorname{Haas}^{20}$ is to calculate the diffusion coefficient from the equation $D=l^{2}$ $\omega / 5.12$, where $\omega$ is the frequency in the transition region on a Nyquist plot where the slope is -2.0 .

The method proposed by Armstrong ${ }^{16}$ extracts the diffusion coefficient from a single frequency and can lead to an error if a datum point does not exist in the transition region with a slope of precisely -2.0 . Here, we develop a model for faradaic impedance of the nickel hydroxide system which is used to obtain the diffusion coefficient at each frequency in the transition region where the slope is finite (lying between -1.5 to -4.5 ). Such a procedure provides more confidence in the value of the diffusion coefficient since it can be calculated at any frequency in the transition region. This method is independent of the charge-transfer kinetics and the value of the diffusion coefficient obtained does not depend on the knowledge of the initial concentration of the mobile species.

The diffusion coefficient of protons decreased from $3.4 \times$ $10^{-8}$ to $3.7 \times 10^{-9} \mathrm{~cm}^{2} \mathrm{~s}^{-1}$ as the state of the electrode changed from fully charged to $30 \%$ SOC. The value of the diffusion coefficient further decreased by another one and a half orders of magnitude to $6.4 \times 10^{-11} \mathrm{~cm}^{2} \mathrm{~s}^{-1}$ at the completely discharged state. These values are higher than those reported from potential step studies by previous investigators. ${ }^{5,6,9,10}$ This may be due to error in the assumption made by those investigators that the initial concentration of protons is equal to the product of the number of nickel sites and the SOC. The functional dependence of the diffusion coefficient with the SOC found in this work suggests that the protons diffuse predominantly through $\mathrm{NiOOH}$ which is interdispersed along with the $\mathrm{Ni}(\mathrm{OH})_{2}$ in the active material.

\section{Mathematical Model}

The faradaic impedance of an electrochemical system is defined as

$$
Z=\frac{d \eta}{d i}
$$

where $\eta$ is the reaction overpotential and $i$ is the resulting reaction current. Assuming that the active material forms a single-phase ideal solid solution, it has been proposed that the current due to reaction 1 is a function of the concentration of protons and the overpotential at the film/electrolyte interface $(y=l),{ }^{7,8,21}$ denoted by $C_{\mathrm{f}}$ and $\eta_{\mathrm{f}}$, respectively. Under these conditions, the total differential of the reaction current can be obtained using the Taylor's series expansion and can be written as

$$
d i=\left(\frac{\partial i}{\partial C_{\mathrm{f}}}\right) d C_{\mathrm{f}}+\left(\frac{\partial i}{\partial \eta_{\mathrm{f}}}\right) d \eta_{\mathrm{f}}
$$

To find an expression for the faradaic impedance in the Laplace domain, $\bar{Z}(s)$, the differential of the overpotential for reaction 1 is divided by the total differential of the reaction current, yielding

$$
\bar{Z}(s)=\left[\frac{\partial \bar{l}}{\partial \bar{C}_{\mathrm{f}}}\left(\frac{d \bar{C}_{\mathrm{f}}}{d \bar{\eta}_{\mathrm{f}}}\right)+\frac{\partial \bar{\imath}}{\partial \bar{\eta}_{\mathrm{f}}}\right]^{-1}
$$

(Overbar indicates variable in the Laplace domain.)

To determine $d \bar{C}_{f} / d \eta$, the concentration profile of the protons is first obtained by solving the one-dimensional time-dependent diffusion equation

$$
\frac{\partial C}{\partial t}=D \frac{\partial^{2} C}{\partial y^{2}}
$$

in the Laplace domain. The initial concentration of protons is assumed to be uniform throughout the film and equal to $C^{0}$, the proton concentration gradient is zero at the conducting substrate/film interface $(y=0)$, and the proton concentration gradient is proportional to the current at the film/electrolyte interface $(y=l)$. The initial and boundary conditions can be mathematically represented as

$$
\begin{array}{ll}
\text { at } t=0, & C=C^{0} \\
\text { at } y=0, & \frac{\partial C}{\partial y}=0
\end{array}
$$

and

$$
\text { at } y=l, \quad D \frac{\partial C}{\partial y}=\frac{-i}{n F A}
$$

The Laplace transformation of Eq. 5 to $6 c$ is taken with respect to $t$ and the resulting ordinary differential equation is solved for $\bar{C}_{f}$, yielding 


$$
\bar{C}_{f}=\frac{C^{0}}{s}-\frac{\bar{i} \operatorname{coth}\left(\sqrt{\frac{s l^{2}}{D}}\right)}{n F A \sqrt{D s}}
$$

Differentiating $\bar{C}_{\mathrm{f}}$ given by Eq. 7 with respect to $\bar{\eta}_{\mathrm{f}}$, substituting the result into Eq. 4 and simplifying yields the expression for the faradaic impedance in the Laplace domain

$$
\bar{Z}(s)=\frac{\partial \bar{\eta}_{\mathrm{f}}}{\partial \bar{\imath}}+\frac{\partial \vec{\imath} / \partial C_{\mathrm{f}}}{\partial \vec{\imath} / \partial \bar{\eta}_{\mathrm{f}}} \frac{1}{n F A \sqrt{D} s}=\operatorname{coth} \sqrt{\frac{s l^{2}}{D}}
$$

To analyze EIS data, Eq. 8 must be transformed from the Laplace domain into the frequency domain. This can be done by substituting the Laplace operator $s$, with a complex variable $p+j \omega{ }^{22}$ (Note: $p$ is the variable that governs the transient response of the electrode in the frequency domain, $j$ is the imaginary number $\sqrt{-1}$, and $\omega$ the frequency in radians per second.) Assuming a small alternating voltage signal $(\leq 10 \mathrm{mV})$ does not perturb the system significantly from the steady state achieved prior to the start of the experiment, $p$ can be set equal to zero and $s=j \omega$ can be substituted into Eq. 8, yielding the expression for the faradaic impedance in the frequency domain

$$
Z(\omega)=\frac{\partial \bar{\eta}_{f}}{\partial \bar{l}}+\frac{(1-j) \sigma}{\sqrt{\omega}} \operatorname{coth}\left((1+j) \sqrt{\frac{\omega l^{2}}{2 D}}\right)
$$

where $\sigma$ (Warburg coefficient) is given by

$$
\sigma=\frac{\partial \vec{i} / \partial C_{\mathrm{f}}}{\partial \bar{\imath} / \partial \bar{\eta}_{\mathrm{f}}} \frac{1}{n F A \sqrt{2 D}}
$$

The faradaic impedance of the nickel hydroxide active material is a linear combination of the charge-transfer resistance and the diffusion impedance (modified Warburg) defined by the first and second terms on the right side of Eq. 9, respectively. To predict the total impedance in the diffusion-controlled regime, the ohmic resistance of the film and the uncompensated solution resistance must be included in series with the faradaic impedance. However, the ohmic resistances in series with the faradaic impedance affect only the magnitude of the impedance and not the slope of the Nyquist plot in the diffusion-controlled regime. In a solid, the time constant for diffusion is much greater than that for capacitance and therefore in the diffusioncontrolled regime, the double-layer capacitance is assumed to be negligible.

To find an expression for the slope in the diffusion-controlled regime on a Nyquist plot, the modified Warburg impedance is separated into real and imaginary components, yielding

$$
Z(\omega)=R e+j I m
$$

where

$$
\begin{aligned}
& \operatorname{Re}=\frac{\sigma}{\sqrt{\omega}}\left\{\frac{\sinh (\psi)-\sin (\psi)}{\cosh (\psi)-\cos (\psi)}\right\} \\
& \operatorname{Im}=\frac{-\sigma}{\sqrt{\omega}}\left\{\frac{\sinh (\psi)+\sin (\psi)}{\cosh (\psi)-\cos (\psi)}\right\}
\end{aligned}
$$

and

$$
\psi=\sqrt{\frac{2 \omega l^{2}}{D}}
$$

The imaginary component of the modified Warburg impedance given by Eq. $10 \mathrm{~b}$ is differentiated with respect to the real component given by Eq. 10a, yielding the expression for the slope

$$
\frac{d I m}{d R e}=-\frac{\left[\frac{C_{1}}{C_{2}}-\frac{S_{1}^{2}}{C_{2}^{2}}-\frac{S_{1}}{\psi C_{2}}\right]}{\left[1-\frac{S_{1} S_{2}}{C_{2}^{2}}-\frac{S_{2}}{\psi C_{2}}\right]}
$$

where $S_{1}, S_{2}, C_{1}$, and $C_{2}$ are as given below

$$
\begin{aligned}
& S_{1}=\sinh (\psi)+\sin (\psi) \\
& S_{2}=\sinh (\psi)-\sin (\psi)
\end{aligned}
$$

$$
\begin{aligned}
& C_{1}=\cosh (\psi)+\cos (\psi) \\
& C_{2}=\cosh (\psi)-\cos (\psi)
\end{aligned}
$$

Equation 11 can be used to extract the diffusion coefficient from the transition region by equating it to the slope in the transition region and solving for $\psi$. The equation reported by Armstrong ${ }^{16}$ for calculating the diffusion coefficient from the transition region can be verified by equating the expression for the slope given by Eq. 11 to -2.0 .

The primary assumption that is implicit in the development of the mathematical model is that migration in the film is negligible and that the only driving force for the transport of protons in the film is a concentration gradient. This implies that the transference number of the electrons $\left(t_{\mathrm{e}}^{-}\right)$is unity and the transference number of the protons $\left(t_{\mathrm{H}}^{+}\right)$ is zero. This assumption is valid if the electronic conductivity of the film is much greater than the ionic conductivity. If the relative conductivities are similar in magnitude then the measured diffusion coefficient is not the diffusion coefficient of protons but a mixed one which is a function of the diffusion coefficient of electrons and protons. Mathias and $\mathrm{Haas}^{20}$ have shown that the maximum error possible in the determination of the mixed diffusion coefficient is a factor of 4.0 when $t_{\mathrm{e}}^{-}$and $t_{\mathrm{H}}^{+}$are both equal to 0.5 . This error is small relative to the nearly three orders of magnitude difference in the diffusion coefficient between the fully charged and discharged states observed here. Literature values for the electronic conductivity as a function of $\mathrm{SOC}^{23,24}$ indicate that the diffusion coefficient measured at SOC greater than $15 \%$ is most likely the result of proton diffusion and therefore the diffusion coefficient reported is that of the protons. There may be an SOC between 15 and $0 \%$ where the diffusion coefficient extracted may be a mixed one which could be overpredicted at most by a factor of four.

\section{Experimental}

An electrochemical quartz crystal nanobalance (EQCN, Elchema Model 501) was used to monitor the mass of the nickel hydroxide film deposited. The active material was deposited by applying a cathodic current of $1 \mathrm{~mA}$ to the working electrode $\left(0.2 \mathrm{~cm}^{2}\right.$ of gold sputtered onto a quartz wafer) of the EQCN (for a complete description of the experimental setup see Ref. 25). The deposition was carried out at room temperature in $1.8 M \mathrm{Ni}\left(\mathrm{NO}_{3}\right)_{2}, 0.175 M$ $\mathrm{Co}\left(\mathrm{NO}_{3}\right)_{2}$, and $0.075 M \mathrm{NaNO}_{3}$ dissolved in a $50 / 50$ volume mixture of water and ethanol. This bath makeup is similar to that used to impregnate commercial nickel electrodes. ${ }^{26}$ A Pine Model AFRDE5 bipotentiostat was used for current control during the deposition. The nickel hydroxide film was rinsed with deionized water, charged at a constant current and conditioned by cycling 20 times at $5 \mathrm{mV} \mathrm{s}^{-1}$ in 3 weight percent $(w / o) \mathrm{KOH}$ solution. The film was then discharged at a constant current of $20 \mu \mathrm{A}$ and the time taken for the discharge was noted. The capacity of the film estimated from the time taken for discharge correlated well with the capacity estimated from the mass of the active material assuming a single electron transfer. The cobalt content of the film was measured by dissolving the film in $2.5 \mathrm{ml}$ of acid and measuring the concentration of the resulting solution with an atomic absorption spectrophotometer. The ratio of nickel to cobalt in the film was 88:12. For impedance studies three different films with masses of 105,85 , and $70 \mu \mathrm{g}$ were deposited. These masses correspond to thicknesses of approximately $1.5,1.2$, and $1.0 \mu \mathrm{m}$ if the density of the active material is assumed to be $3.5 \mathrm{~g} \mathrm{~cm}^{-3}{ }^{27}$

An EG\&G Princeton Applied Research (PAR) Model 388 impedance system was used for all EIS experiments. The system consists of a Model 273 potentiostat/galvanostat, a Model 5208EC Lockin analyzer, and a personal computer. This system is capable of measuring the electrochemical impedance over a frequency range of $100 \mathrm{kHz}$ to $0.05 \mathrm{mHz}$. Impedance measurements were made at various SOC for the three different film thicknesses. Here, the SOC is defined with respect to the total useful capacity, determined from the time taken for discharge at a constant current of 


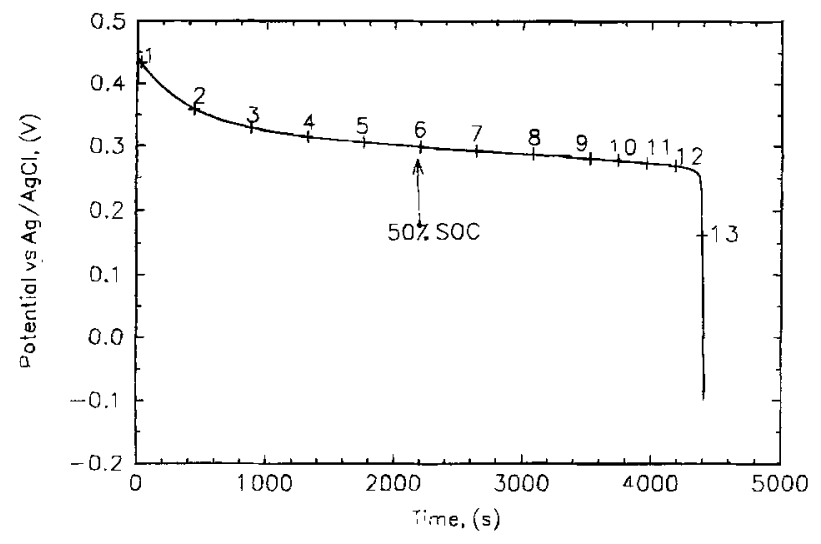

Fig. 2. Experimental discharge curve obrained for a $1.5 \mu \mathrm{m}$ film at a constant current of $20 \mu \mathrm{A}$. Each point on the discharge curve corresponds to an SOC that can be determined by dividing the corresponding time on the ordinate with the total time for discharge. For example $50 \% \mathrm{SOC}$ is indicated on the figure.

$20 \mu \mathrm{A}$. Figure 2 illustrates an experimental discharge curve obtained by discharging the $1.5 \mu \mathrm{m}$ film at a constant current of $20 \mu \mathrm{A}$. The total discharge time is $4400 \mathrm{~s}$ as shown in Fig. 2. Each point (1 to 13) marked on the discharge curve stands for a particular SOC at which impedance measurements were made. The active material was discharged for a fixed time at the same current and the SOC determined from the ratio of the time discharged to the time required for complete discharge. For example, a 2200 s discharge (point number 6 on Fig. 2) corresponds to $50 \%$ SOC for the $1.5 \mu \mathrm{m}$ film. After the film was discharged to a particular SOC, the electrode was left on open circuit until a stable potential was observed (5-15 $\mathrm{min}$ ) before an experiment was conducted. After data were collected at a particular SOC, the electrode was charged again, cycled twice at $5 \mathrm{mV} \mathrm{s}^{-1}$ and discharged to the next SOC. All EIS experiments were run at open circuit using an alternating voltage signal of $10 \mathrm{mV}$ (root mean square). The electrode was stable during the measurements and its open-circuit potential changed by $1 \mathrm{mV}$ or less.

At high states of charge ( $>30 \%$ ), five sets of experiments were conducted at each SOC. At low states of charge $(<30 \%)$, owing to the necessity of making measurements at low frequencies and therefore over long periods of time, only two (one in the fully discharged state) could be collected before the open-circuit potential of the electrode changed by $1 \mathrm{mV}$ due to self-discharge. ${ }^{28}$ The EIS data at a given SOC were highly reproducible. The multiple data sets at each SOC were averaged prior to calculating the diffusion coefficients. EIS data were highly reproducible for two different films with a thickness of $1.5 \mu \mathrm{m}$.

\section{Results and Discussion}

Simulation results. - The effect of varying the diffusion coefficient and the thickness of the film on the diffusioncontrolled Nyquist plane behavior is simulated using Eq. 9 and illustrated in Fig. 3a and b, respectively. Several investigators ${ }^{11-20}$ have discussed the behavior of the modified Warburg impedance and reported that it is characterized by three distinct regions, viz. the semi-infinite diffusion, transition, and finite diffusion regions. The frequency ranges in which the three regions are observed is controlled by the values of the diffusion coefficient and the thickness of the film. At high frequencies when $\omega \gg D / l^{2}$, the hyperbolic cotangent term tends to unity, the modified Warburg impedance behaves like the conventional Warburg impedance, and a straight line region with a slope of $45^{\circ}$ is observed on a Nyquist plot. At lower frequencies, when $\omega \ll D / l^{2}$ the phase angle tends to infinity and a vertical line region perpendicular to the real axis is seen on a Nyquist plot. In this region, the diffusion behavior is limited by the finite length of the film and a capacitive effect is observed on the Nyquist plot due to charge saturation. ${ }^{19}$
At intermediate frequencies when $\omega \sim D / l^{2}$, a transition from the semi-infinite diffusion region to the finite diffusion region is seen. The region between the semi-infinite diffusion region and the finite diffusion region is called the transition region. ${ }^{16}$

Figure 3a shows Nyquist plot simulations for $D=1.0 \times$ $10^{-9}$ and $3.0 \times 10^{-9} \mathrm{~cm}^{2} \mathrm{~s}^{-1}$ and $l=1.5 \mu \mathrm{m}$ and Fig. $3 \mathrm{~b}$ contains simulations for $D=1.0 \times 10^{-9}$ and $l=1.0,1.2$, and $1.5 \mu \mathrm{m}$. For illustration, $\sigma$ and the charge-transfer resist ance in Eq. 9 were set equal to $5.0 \Omega / \mathrm{s}^{1 / 2}$ and $22.0 \Omega$, respectively, for all the simulations presented here. The chargetransfer resistance and $\sigma$ affect only the magnitude of the impedance and not the slope of the Nyquist plot in the frequency range where diffusion is the rate-limiting process. We can see from Fig. $3 a$ that both the Nyquist plots exhibit each of the three characteristic regions and that the onset of the transition region is observed earlier (at higher frequencies) as the value of the diffusion coefficient increases. From Fig. 3b, we can observe that the increase in the film thickness has the same effect as a decrease in the diffusion coefficient. Therefore, if the diffusion coefficient of protons decreases as a function of SOC, the onset of the transition region must be observed at progressively lower frequencies as the electrode is discharged. At any SOC, the onset of the transition region must be observed at higher frequencies as the thickness of the film is decreased. For example, for the $1.0 \mu \mathrm{m}$ film, the onset of the transition region must be observed at a frequency which is approximately 2.25 times the corresponding frequency for the $1.5 \mu \mathrm{m}$ film.

Experimental results.-Figure 4 shows the Nyquist plot obtained for the $1.5 \mu \mathrm{m}$ thick film, in the completely discharged state (point number 13 on Fig. 2), over a frequency range of $100 \mathrm{kHz}$ to $1.5 \mathrm{mHz}$. At high frequencies the semicircle is characteristic of the charge-transfer resistance acting in parallel with the double-layer capacitance. At intermediate frequencies $(0.085-0.0075 \mathrm{~Hz})$ a straight line with a slope of $\sim 45^{\circ}$ is seen, characteristic of the semi-infinite diffusion. At still lower frequencies $(<1.5 \mathrm{mHz})$, the finite length effects are observed, and there is a transition from the $45^{\circ}$ line toward a vertical line through the transition region which lies in a frequency range of 6.5 to $2.5 \mathrm{mHz}$. In the finite diffusion region, it was only possible to get two data points owing to the need for extremely time-consuming low frequency measurements. The transition region for a fully discharged film was observed in a frequency range which was approximately three orders of magnitude less than the corresponding range for the completely charged film. Impedance measurements at all other states of charge were made only over a limited range of frequencies in which diffusion control was observed and thus the data presented at the other SOC (Fig. 5a-b, 6) do not contain the semicircle.

Figure 5a shows Nyquist plots at 80 and $60 \%$ SOC and Fig. $5 \mathrm{~b}$ shows the Nyquist plots obtained for 50 and $30 \%$ SOC over the frequency range in which diffusion dominates. The frequency range over which impedance measurements were made for the 80 and $60 \%$ SOC was $0.3-11.9$ and $0.15-5.65 \mathrm{~Hz}$, respectively. For the 50 and $30 \% \mathrm{SOC}$, the corresponding frequency ranges are 0.05-5.65 and 0.03$1.13 \mathrm{~Hz}$, respectively. The frequency at which the onset of the transition region is observed is marked on the plots. It can be seen that the onset of the transition region shifts to lower frequencies at lower SOC indicating that the diffusion coefficient is steadily decreasing with decreasing SOC. Figure 6 shows Nyquist plots at $40 \%$ SOC for three different films $1.5,1.2$, and $1.0 \mu \mathrm{m}$ thick. The frequency range over which impedance measurements were made for all. three thicknesses is the same $(0.09-3.4 \mathrm{~Hz})$. The frequencies at which the onset of the transition region was observed is also marked on the plot.

The diffusion coefficient at all SOC was determined at each frequency in the transition region (typically 3-5 points) by performing a least squares fit of the data to the exponential function

$$
\text { Im }=a \exp (b R e)
$$



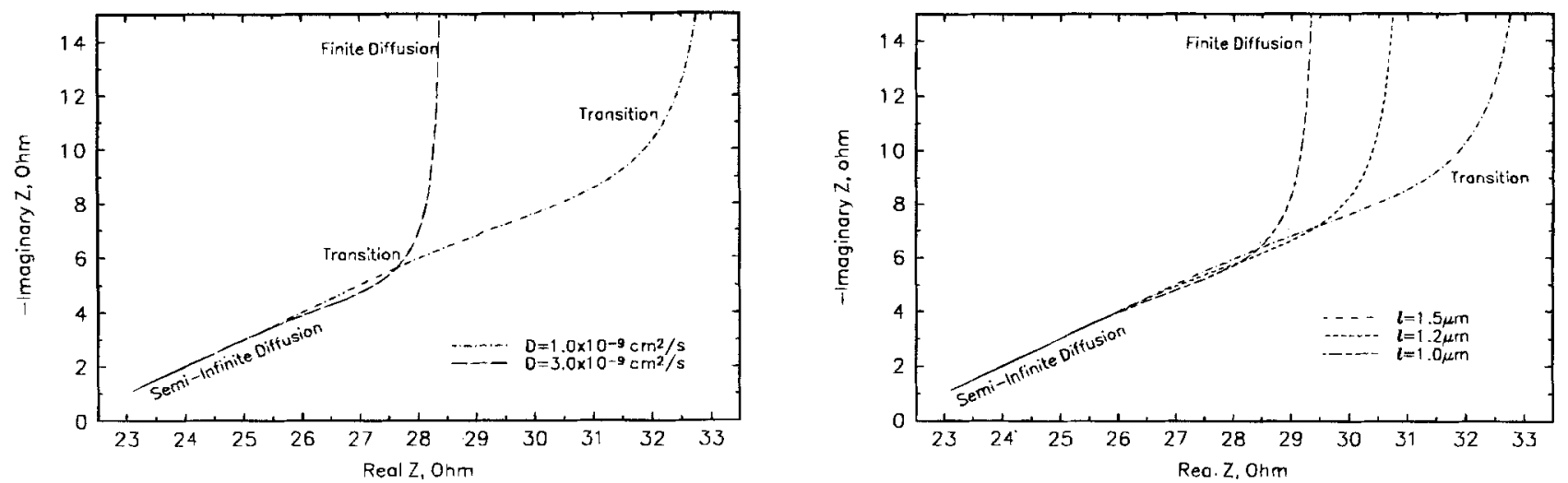

Fig. 3. (a, leff) Simulated Nyquist plots for a $1.5 \mu \mathrm{m}$ film, for $D=1.0 \times 10^{-9}$ and $3.0 \times 10^{-9} \mathrm{~cm}^{2} \mathrm{~s}^{-1}$ over a frequency range of 0.016 to $3.18 \mathrm{~Hz}$. The onset of the transition region is observed at progressively higher frequencies as the diffusion coefficient increases. (b, right) Simulated Nyquist plots for three different film thicknesses of $1.5,1.2$, and $1.0 \mu \mathrm{m}$ for $D=1.0 \times 10^{-9} \mathrm{~cm}^{2} \mathrm{~s}^{-1}$ over a frequency range of 0.016 to $3.18 \mathrm{~Hz}$. The onset of the transition region is observed at progressively lower frequencies as the thickness of the film increases from 1.0 to $1.5 \mu \mathrm{m}$. For illustration, $\sigma$ and the charge-transfer resistance were set equal to $5.0 \Omega / \mathrm{s}^{1 / 2}$ and $22 \Omega$, respectively. The semi-infinite diffusion, finite diffusion, and the transition regions are depicted on the plots.

where $a$ and $b$ are fitting parameters. Equation 12 was used to fit the data presented in Fig. 5a-b and 6 . It can be seen from the figures that the equation fits the data well. After the fitting parameters were determined from regression analysis, the function was differentiated at each frequency to obtain the slope. The slope at each frequency was used in conjunction with Eq. 11 to solve for $\psi$. The diffusion coefficient was then obtained from the definition of $\psi$ (given by Eq. 10c) at the corresponding frequency and film thickness. The diffusion coefficients reported here were obtained by averaging the values calculated at each frequency in the transition region. The value calculated at different frequencies in the transition region varied by less than $20 \%$.

Figure 7 summarizes the variation of the diffusion coefficient of protons as a function of SOC. The value of the diffusion coefficient decreased rapidly near the end of discharge $(<20 \%$ SOC) necessitating the collection of impedance data at every $5 \%$ SOC intervals. The value of the diffusion coefficient for $\mathrm{NiOOH}$ (point number 1 on Fig. 2) was $3.4 \times 10^{-8}$ and for $\mathrm{Ni}(\mathrm{OH})_{2}$ (point number 13 on Fig. 2) the value was $6.4 \times 10^{-11} \mathrm{~cm}^{2} \mathrm{~s}^{-1}$. Figure 7 also contains a plot of the function proposed by Bouet et al. ${ }^{29}$

$$
D=D_{1}\left[\theta+\left(\frac{D_{2}}{D_{1}}\right)^{1 / 2}(1-\theta)\right]^{2}
$$

where $\theta$ is the SOC or the fraction of $\mathrm{NiOOH}$ present in the solid solution, $D_{1}$ the diffusion coefficient of the protons in pure NiOOH $\left(3.4 \times 10^{-8} \mathrm{~cm}^{2} \mathrm{~s}^{-1}\right.$ for $\left.l=1.5 \mu \mathrm{m}\right)$, and $D_{2}$ the diffusion coefficient of protons in pure $\mathrm{Ni}(\mathrm{OH})_{2}(6.4 \times$

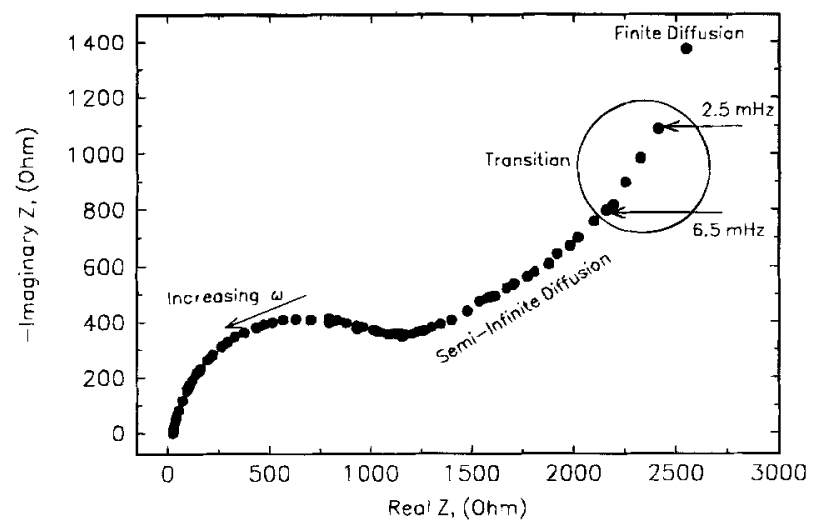

Fig. 4. Nyquist plot for a $1.5 \mu \mathrm{m}$ film in the completely discharged state (point number 13 on Fig. 2) over a frequency range of $100 \mathrm{kHz}$ to $1.5 \mathrm{mHz}$. The transition region occurs in a frequency range of 6.5 to $2.5 \mathrm{mHz}$. The semicircle seen at high frequencies is due to the parallel combination of the charge-transfer resistance and the double-layer capacitance.
$10^{-11} \mathrm{~cm}^{2} \mathrm{~s}^{-1}$ for $\left.l=1.5 \mu \mathrm{m}\right)$. Equation 13 is a mixing rule in terms of the root mean square displacement of the diffusing species in a solid solution comprised of a homogeneous mixture of two solids. As can be seen from Fig. 7, Eq. 13 fits all the data to within $10 \%$

The following three mechanisms are considered to explain the functionality of the diffusion coefficient with SOC.

1. The crystal structure of the film changes such that the lattice spacing available for the diffusion of protons decreases as the electrode is discharged.

2. The diffusion coefficient is constant, but the diffusion length ( $l$ used in Eq. 10c for the calculation of $D$ ) changes as a function of SOC due to a moving reaction plane.

3 . Protons move predominantly through the oxidized $\mathrm{NiOOH}$ phase which contains a defect concentration which is orders of magnitude greater than the defect concentration in the reduced $\mathrm{Ni}(\mathrm{OH})_{2}$ phase, such that the measured $D$ is an effective diffusion coefficient depending on the fraction of the oxidized phase available for diffusion.

The diffusion coefficient would decrease as the electrode is discharged if there was a corresponding decrease in the crystal lattice spacing of the active material. Nickel hydroxide $\left[\mathrm{Ni}(\mathrm{OH})_{2}\right]$ and nickel oxyhydroxide $[\mathrm{NiOOH}]$ have a layer type configuration in which slabs of $\mathrm{NiO}_{2}$ are separated by galleries containing protons. ${ }^{30}$ It is known that $\mathrm{Ni}(\mathrm{OH})_{2}$ exists as two structural modifications called the $\alpha$ and the $\beta$ forms. ${ }^{31}$ The $\alpha$ form of $\mathrm{Ni}(\mathrm{OH})_{2}$ is comprised of galleries containing water and other cations in addition to protons. The $\alpha-\mathrm{Ni}(\mathrm{OH})_{2}$ stabilizes by dehydrating to the more stable $\beta$ form on cycling or on standing in alkali with a resultant decrease in lattice spacing. The $\beta-\mathrm{Ni}(\mathrm{OH})_{2}$ can be oxidized to $\beta-\mathrm{NiOOH}$ on charging and to $\gamma-\mathrm{NiOOH}$ on overcharge. Even though the electrochemical deposition results in the formation of $\alpha-\mathrm{Ni}(\mathrm{OH})_{2}$, the procedure used for conditioning of the films prior to EIS experiments most likely results in the transformation of $\beta-\mathrm{Ni}(\mathrm{OH})_{2}$ to $\beta$ NiOOH. ${ }^{1,9,31}$ MacArthur $^{1}$ showed that a transformation between the $\alpha-\mathrm{Ni}(\mathrm{OH})_{2}$ and $\gamma-\mathrm{NiOOH}$ phases of the active material results in two distinct anodic peaks during cyclic voltammetry. In this work no peak splitting was observed while cycling the film during or after conditioning. This is perhaps due to the presence of Co in the film which acts as a $\beta$-phase stabilizer and results in the film being cycled between the $\beta-\mathrm{Ni}(\mathrm{OH})_{2}$ and $\beta-\mathrm{NiOOH}$ phases. ${ }^{32}$ Briggs et $a l^{33}$ and Kober ${ }^{34}$ showed using $\mathrm{x}$-ray measurements and infrared (IR) observations, respectively, that the lattice parameters of the $\beta-\mathrm{Ni}(\mathrm{OH})_{2}$ and $\mathrm{NiOOH}$ are almost identical. This suggests that the approximately three orders of magnitude change in the value of the diffusion coefficient measured is not due to the change in the crystal structure.

A moving reaction boundary model during charge and discharge has been proposed in the literature ${ }^{30}$ and suggests 

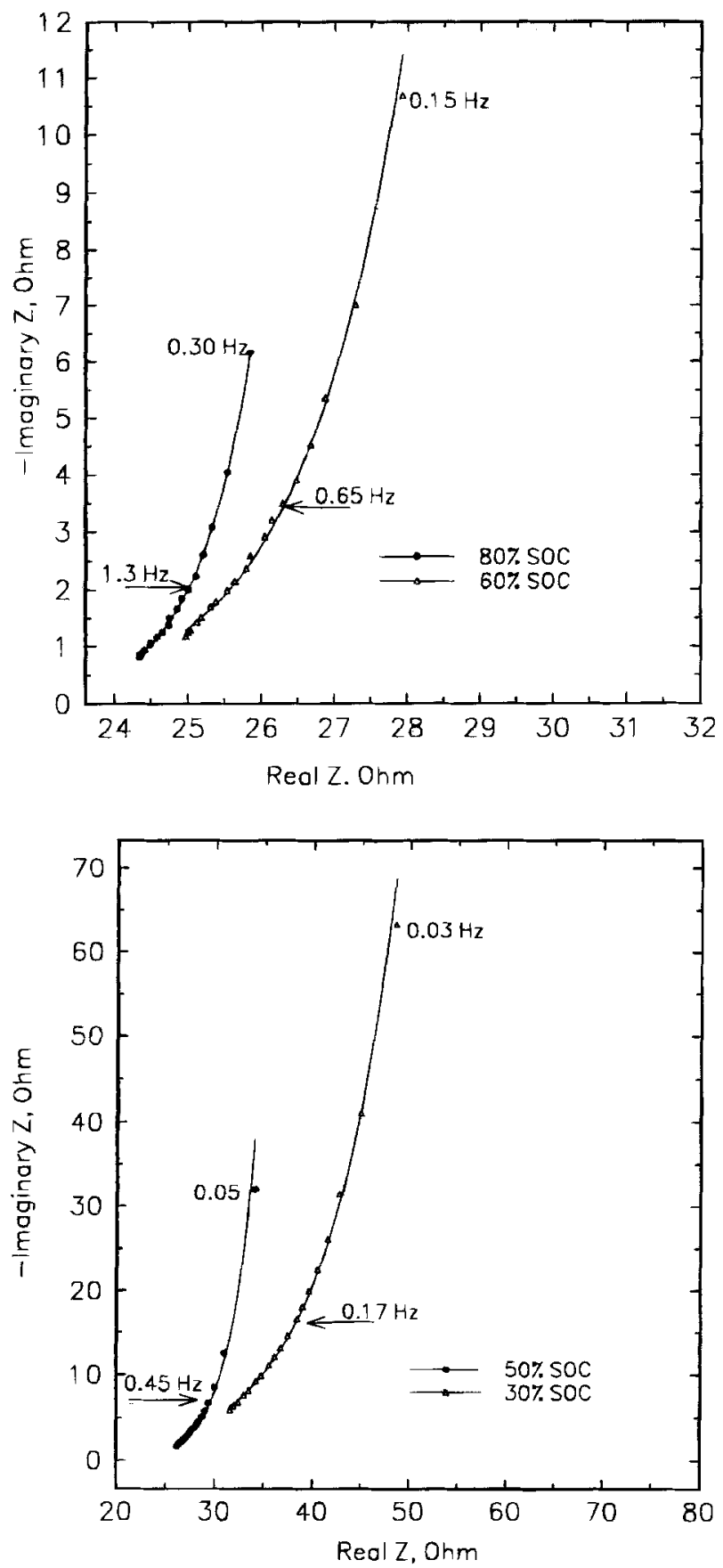

Fig. 5. (a, top) Nyquist plot for a $1.5 \mu \mathrm{m}$ film at 80 and $60 \%$ states of charge over a frequency range of $0.3-11.9$ and $0.15-5.65 \mathrm{~Hz}$, respectively. The frequencies at which the onset of the transition region for the 80 and $60 \%$ states of charge is observed is 1.3 and $0.65 \mathrm{~Hz}$, respectively. (b, bottom) Nyquist plot for a $1.5 \mu \mathrm{m}$ film at 50 and $30 \%$ states of charge over a frequency range of 0.05-5.6 and 0.03-1.1 Hz, respectively. The frequency at which the onset of the transition region for the 50 and $30 \%$ states of charge is seen is 0.45 and $0.17 \mathrm{~Hz}$, respectively. The data is fit to Eq. 12. The onset of the transition region shifts to lower frequencies as the SOC decreases indicating that the diffusion coefficient is decreasing as a function of SOC.

that the diffusion length of the protons is changing as a function of SOC. The active material in the reduced form $\left[\mathrm{Ni}(\mathrm{OH})_{2}\right]$ is pale green in color and is black in the oxidized form $[\mathrm{NiOOH}]$, and some researchers have observed the motion of a color boundary during the charge and discharge of the active material. ${ }^{35,36}$ Huggins et al. ${ }^{30}$ attribute this moving color boundary to the movement of the $\mathrm{NiOOH} / \mathrm{Ni}(\mathrm{OH})_{2}$ interface. According to the authors, the charge/discharge reaction takes place at the NiOOH/ $\mathrm{Ni}(\mathrm{OH})_{2}$ boundary (i.e., reaction plane) and this boundary

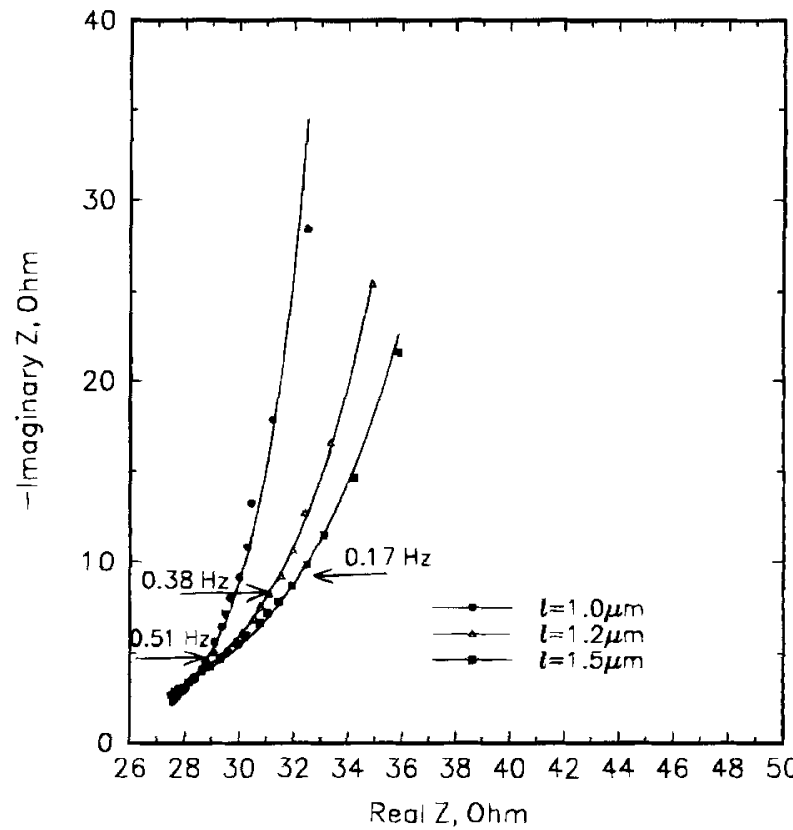

Fig. 6. Nyquist plots for three different thicknesses of 1.5, 1.2, and $1.0 \mu \mathrm{m}$ at $40 \% \mathrm{SOC}$. The onset of the transition region is seen at 0.27 , 0.38 , and $0.51 \mathrm{~Hz}$, respectively. The data is fit to $\mathrm{Eg}$. 12. The onset of the transiton region is observed at progressively higher frequencies as the film thickness decreases.

moves from the film/electrolyte interface toward the conducting substrate/film interface during discharge and vice versa during charge. If the notion of a sharp moving reaction plane is true, the value of the diffusion length in the transition region is not equal to the thickness of the film but is a function of the SOC given by

$$
\delta=l(1-\theta)
$$

where $\delta$ is the distance from the reaction plane to the film/ electrolyte interface. Therefore $\delta$ will be equal to the thickness of the film at $0 \%$ SOC and equal to zero when the electrode is fully charged. It is possible to obtain the value of $D / \delta^{2}$ at all states of charge by dividing $D_{2}$ (diffusion coefficient in the completely discharged state), by the square of the right side of Eq. 14

In Fig. 8, the $D / \delta^{2}$ data obtained for the $1.5 \mu \mathrm{m}$ film is plotted along with the corresponding fit (curve a) utilizing Eq. 13 (with $l=\delta$ ). Also plotted on Fig. 8 , is $D / \delta^{2}$ for a moving reaction plane where $D=D_{2}$ and $\delta=l(1-\theta)$ (curve b). (Note: $\theta$ in the completely charged state was assumed to

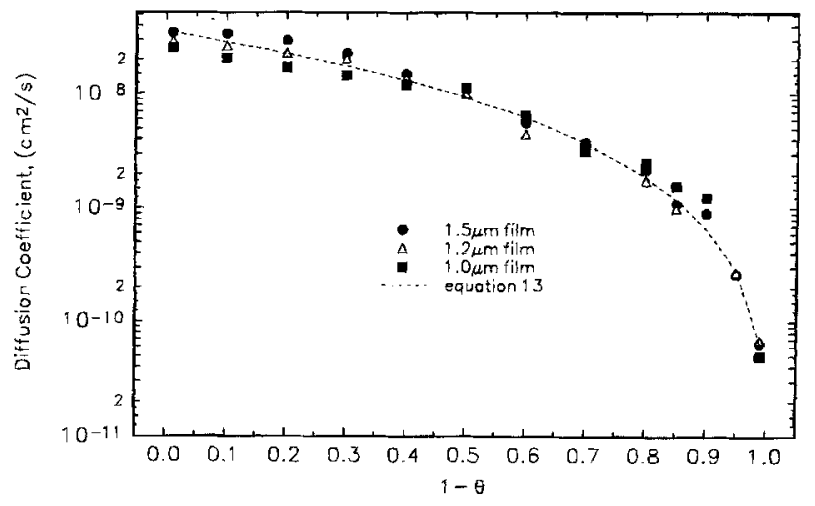

Fig. 7. Diffusion coefficient of protons in the nickel hydroxide film as a function of SOC. The diffusion coefficient of protons decreases approximately three orders of magnitude as the electrode changes from the completely charged to the completely discharged state. Equation 13 is plotted along with the data. 


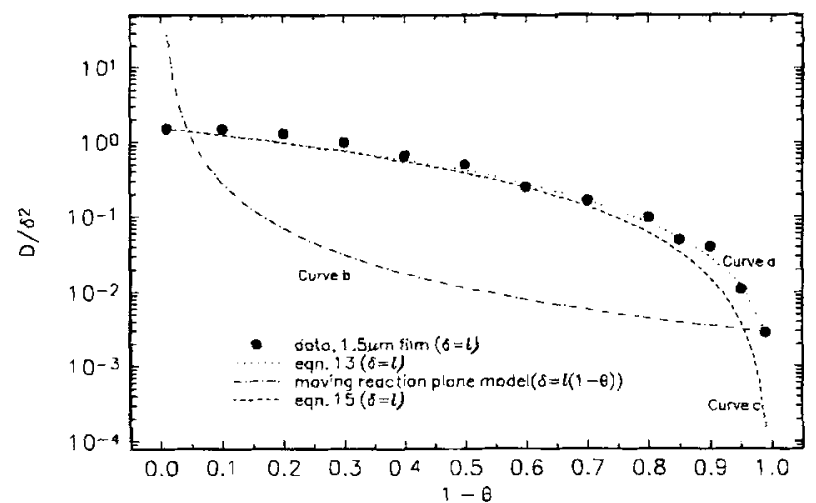

Fig. 8. $D / \delta^{2}$ data, $\delta=\|$ obtained for the $1.5 \mu \mathrm{m}$ film is shown. Curves $a$ and $c$ on the figure correspond to the plots of Eq. 13 and 15 , respectively. Also shown on the plot is curve c, which is $D / \delta^{2}$ calculated assuming $\delta$ to be a linear function of the SOC (reaction plane model). It can be seen from the figure that, curves $a$ and $c$ fit the data well, whereas curve $b$ does not match the qualitative trend exhibited by the data.

be 0.99 .) It can be seen from the figure that curve a fits the data, but curve b does not show the same qualitative trend. This suggests that the moving color boundary seen during charge and discharge of the electrode is not due to the movement of the reaction plane. One explanation for the observed movement of a color boundary could be based on the fact that, at high to moderate charge and discharge rates, there is a gradient in the SOC within the film due to the diffusion limitation of protons. ${ }^{8}$

As described above, the structure of the $\mathrm{NiOOH} / \mathrm{Ni}(\mathrm{OH})_{2}$ active material consists of layers of $\mathrm{NiO}_{2}$ separated by proton containing galleries. ${ }^{30} \mathrm{NiOOH}$ has been described as a proton deficient phase in which $50 \%$ of proton gallery sites are vacant. ${ }^{3,4}$ The proton sites in $\mathrm{Ni}(\mathrm{OH})_{2}$ are fully occupied. If proton diffusion takes place by a hopping process, whereby the protons move from one site to an adjoining vacancy, the diffusion coefficient depends on the concentration of protons and vacancies. A sharp drop in the diffusion coefficient should occur when either of the concentrations approaches zero. In nickel hydroxide, at low states of charge the diffusion coefficient of protons decreases sharply because the vacancy concentration approaches zero. No sharp drop in the diffusion coefficient of protons was observed at high states of charge because the product of the proton and vacancy concentration is at a maximum when the average valence state of nickel in the active material is approximately +3 . A sharp drop in the proton concentration is not expected until the valence state of nickel approaches +4 . Although valence states greater than +3 are believed to occur, it is not expected that an average valence state of +4 was reached during our experimentation.

In Fig. 7 and 8 we illustrate that the data can be fit using a mixing rule in terms of the root mean square of displacement of the diffusing species (see Eq. 13). The good fit obtained using Eq. 13 indicates that the film is most likely comprised of a homogeneous mixture of $\mathrm{NiOOH}$ and $\mathrm{Ni}(\mathrm{OH})_{2}$. As discharge proceeds the mole fraction of $\mathrm{Ni}(\mathrm{OH})_{2}$ increases, effectively the proton vacancy concentration of the solid decreases, resulting in a solid with a decreased diffusion coefficient.

Since the proton diffusion coefficient in the reduced phase is three orders of magnitude less than that of the oxidized phase (i.e., $D_{2}$ in Eq. 13 is significantly less than $D_{1}$ ), the active material alternatively can be considered analogous to a porous electrode in which diffusion proceeds only through one phase. Equation 13 can be reduced to the following by setting $D_{2}$ to zero

$$
D=D_{1} \theta^{2}
$$

Equation 15 is similar to the expression Newman $^{37}$ reported for the diffusion of an ion in the solution phase of a porous electrode, with the SOC being analogous to the porosity. Curve $\mathrm{c}$ in Fig. 8 is a plot of Eq. 15 ( $\theta$ in the completely discharged state was assumed to be 0.01 ). As expected the fit is similar to that of Eq. 13 (curve a). The fit obtained by Eq. 15 is more sensitive to the SOC than that obtained using Eq. 13 and so digresses from the data toward the end of discharge to a greater degree. However, if the data points in the fully reduced state actually corresponded to $3 \%$ SOC (which is plausible), the fit would be virtually indistinguishable from that obtained using Eq. 13.

\section{Conclusion}

A model for the complex faradaic impedance of the nickel hydroxide electrode was developed and was used to extract the diffusion coefficient of protons as a function of SOC and thickness of the film from electrochemical impedance spectroscopy data. All the data in the transition region of the Nyquist plot can be analyzed using the expression for the slope of the Nyquist plot developed to extract a constant diffusion coefficient. The diffusion coefficient of protons in the $\beta$-phase of the active material decreased by approximately three orders of magnitude as the electrode changed from the completely charged to the completely discharged state. The three orders of magnitude decrease in the diffusion coefficient of protons can be explained on the assumption that the protons move predominantly through the oxidized phase [NiOOH] which is interdispersed along with the reduced phase $\left[\mathrm{Ni}(\mathrm{OH})_{2}\right]$ in the active material.

\section{Acknowledgment}

The authors acknowledge the financial support from the Office of Research and Development of the U.S. Central Intelligence Agency for this project. The authors also acknowledge useful discussions with Dr. Mark Mathias of Mobil R\&D. and Ms. Pauline De' Vidts at the University of South Carolina.

Manuscript submitted Sept. 6, 1994; revised manuscript received Dec. 28, 1994

University of South Carolina assisted in meeting the publication costs of this article.

\section{LIST OF SYMBOLS}

$A$ area of the nickel hydroxide film, $0.2 \mathrm{~cm}^{2}$

C proton concentration, $\mathrm{mol} \mathrm{cm}^{-3}$

$C^{0}$ initial proton concentration, $\mathrm{mol} \mathrm{cm} \mathrm{cm}^{-3}$

$D$ diffusion coefficient of protons, $\mathrm{cm}^{2} \mathrm{~s}^{-1}$.

$D_{1} \quad D$ in $\mathrm{NiOOH}, \mathrm{cm}^{2} \mathrm{~s}^{-1}$.

$D_{2} \quad D$ in $\mathrm{Ni}(\mathrm{OH})_{2}, \mathrm{~cm}^{2} \mathrm{~s}^{-i}$

$F$ Faraday's constant, $96487 \mathrm{C} / \mathrm{equiv}$

$i \quad$ Faradaic current, $\mathrm{A}$

Im imaginary impedance, $\Omega$

j Imaginary number, $\sqrt{-1}$

thickness of film, cm

$n$ number of electrons taking part in reaction 1,1

$R e$ Real impedance, $\Omega$

$s \quad$ Laplace frequency, $\mathrm{s}^{-1}$

$t$ time, $s$

$y$ distance into hydroxide film, $\mathrm{cm}$

$Z$ complex impedance, $\Omega$

Greek

$\theta \quad$ state of charge

$\omega$ angular frequency, rad s $^{-1}$

$\eta$ overpotential, $V$

$\hat{\delta}$ distance of the reaction plane from the film/electrolyte interface, $\mathrm{cm}$

$\psi \quad \sqrt{\frac{2 \omega l^{2}}{D}}$

$\sigma \quad$ Warburg coefficient, $\Omega / \mathrm{s}^{1 / 2}$

Subscript

f film/electrolyte interface at $y=l$

\section{REFERENCES}

1. D. M. MacArthur, This Journal, 117, 422 (1970).

2. A. H. Zimmerman and P. K. Effa, ibid., 129, 983 (1984).

3. Z. Takehara, M. Kato, and S. Yoshizawa, Electrochim. Acta, 16, 833 (1971).

4. P. D. Lukovtsev and G. J. Slaidin, ibid., 6, 17 (1962). 
5. G. W. D. Briggs and P. R. Snodin, ibid., 27, 565 (1982)

6. C. Zhang and S. Park, This Journal, 134, 2966 (1987).

7. Z. Mao, P. De Vidts, R.E. White, and J. Newman, ibid., 141, 54 (1994).

8. J. W. Weidner and P. Timmerman, ibid., 141, 346 (1994).

9. D. M. MacArthur, ibid., 117, 729 (1970).

10. D. Fan, Ph.D. Thesis, Texas A\&M University, College Station, TX (1991).

11. C. Ho, I. D. Raistrick, and R. A. Huggins, This Journal, 127, 343 (1980).

12. R. D. Armstrong, B. Lindhom, and M. Sharp, J. Electroanal. Chem., 202, 69 (1986).

13. J. P. Randin and R. Viennet, This Journal, 129, 2349 (1982).

14. J. Farcy, R. Messina, and J. Perichon, ibid., 137, 1337 (1990).

15. R. Cabanel, G. Barral, J. P. Diard, B. Le Gorrec, and C. Montella, J. Appl. Electrochem., 23, 93, (1993).

16. R. D. Armstrong, J. Electroanal. Chem., 198, 177 (1986).

17. G. Lang and G. Inzelt, Electrochim. Acta, 36, 847, (1991).

18. I. Rubinstein, J. Risphon, and S. Gottesfeld, This Journal, 133, 729, (1986).

19. T. B. Hunter, P. S. Tyler, W. H. Smyrl, and H. S. White, ibid., 134, 2198, (1987).

20. M. F. Mathias and O. Haas, J. Phys. Chem., 96, 3174 (1992).

21. D. Fan and R. E. White, This Journal., 138, 2952 (1991).

22. B. A. Boukamp and G. A. Wiegers, Solid State Ionics, 9\&10, 1193, (1983)

23. P. A. Antonenko, V. Z. Barusukov, N. G. Krapivnii, and L. N. Sagoyan, Vopr. Khim. Khim. Teknol. (Kharkov),
25,135 (1972)

24. M. J. Natan, D. Belanger, M. K. Carpenter, and M. S. Wrighton, J. Phys. Chem., 91, 1834 (1987).

25. C. C. Streinz, A. P. Hartman, S. Motupally, and J. W. Weidner, This Journal, 142, 1084 (1995).

26. B. A. Johnson, R. E. Ferro, G. M. Swain, and B. J Tatarchuk, J. Power Sources, 47, 251 (1994).

27. A. H. Zimmerman and P. K. Effa, Abstract 28, p. 43, The Electrochemical Society Extended Abstracts, Vol. 85-2, Las Vegas, NV, Oct. 13-18, 1985.

28. B. E. Conway and P. L. Bourgalt, Trans. Faraday Soc., $\mathbf{5 8}, 593,(1962)$

29. J. Bouet, F. Richard, and P. Blanchard, in Proceedings of the Symposium on Nickel Hydroxide Electrodes, D. A. Corrigan and A. H. Zimmerman, Editors, PV 90-4, p. 260, The Electrochemical Society Proceedings Series, Pennington, NJ (1990).

30. R. A. Huggins, M. Wohlfahrt-Mehrens, and L. Jorinssen, Mat. Res. Soc. Symp. Proc., 293, 57 (1993).

31. H. Bode, K. Dehmelt, and J. Witte, Electrochim. Acta., 11, 1079 (1966).

32. O. G. Malandin, A. V. Vasev, P. N. Bityutskii, I. S. Shamina, and G. V. Suchkova, Elektrokhimiya, 14, 91 (1978).

33. G. W. D. Briggs, E. Jones, and W. F. K. Wynne-Jones, Trans. Faraday Soc., 51, 394 (1955).

34. F. P. Kober, This Journal, 112, 1064 (1965).

35. R. Barnard, C. F. Randell, and F. L. Tye, J. Appl. Electrochem., 10, 109, (1980).

36. G. W. D. Briggs and M. Fleischmann, Trans. Faraday Soc., 67, 2397, (1971).

37. J. Newman, Electrochemical Systems, Prentice Hall Inc., Englewood Cliffs, NJ (1973). 